\title{
SUPERCONDUCTING RF CONTROL ISSUES AT CESR*
}

\author{
S. Belomestnykh ${ }^{\dagger}$, S. Henderson, R. Kaplan \\ Laboratory of Nuclear Studies, Cornell University, Ithaca, NY 14853, USA
}

\begin{abstract}
RF controls of the superconducting cavities have to deal with an extremely heavy beam loading in CESR. A feature of the CESR RF system operation is that superconducting cavities are under-coupled at high beam currents. This means that the RF system operates very close to Robinson stability limit. Analysis of the steadystate stability of the CESR RF system and the results of measurements with beam are presented. Two more SRF cavities will be installed in CESR for short bunch operation. This will tighten tolerances to RF signal regulation errors. New control electronics is under development to meet the challenge.
\end{abstract}

\section{INTRODUCTION}

Electron-positron collider CESR operates at a beam energy of $5.3 \mathrm{GeV}$ with the total current up to $780 \mathrm{~mA}$ in two beams [1]. This beam current presents heavy beam loading to the superconducting RF system [2]. A feature of present operation is that superconducting cavities are under-coupled at high beam currents. This means that the RF system operates very close to Robinson stability limit. Analysis of the steady-state stability of the CESR RF system is presented alongside with some other aspects of the RF system controls. Also discussed are the RF control issues of the recently proposed upgrade of CESR for lowenergy operation, CESRc [3].

\section{STEADY-STATE STABILITY}

The ratio of the beam-induced voltage $\left(V_{\mathrm{br}}\right)$ on resonance to the cavity voltage $\left(V_{\mathrm{c}}\right), Y=V_{\mathrm{br}} / V_{\mathrm{c}}$, is the measure of the beam loading. High intensity colliders ("factories") are typically operating in the beam loading parameter range of $Y=2 \ldots 5$ [4] with very small stability margin.

Single-cell superconducting cavities [5] have been designed with a fixed RF coupler to match 1 A CESR beam current at accelerating gradient of $10 \mathrm{MV} / \mathrm{m}$. So long as present operating gradient is $6.2 \mathrm{MV} / \mathrm{m}$, cavities become under-coupled for beam currents above

$$
I_{0} \cong \frac{V_{c}}{R / Q \cdot Q_{l} \cdot \cos \varphi_{S}}=500 \mathrm{~mA},
$$

where $R / Q=89 \Omega$ is the cavity shunt impedance, $Q_{l}=$ $2.4 \times 10^{5}$ is the loaded quality factor (it was $2 \times 10^{5}$ in the original design [5]), and $\varphi_{\mathrm{S}} \cong 80^{\circ}$ is the synchronous phase measured from the RF wave crest. This means that

\footnotetext{
*Work supported by the National Science Foundation.

${ }^{\dagger}$ sab@lns.cornell.edu
}

i) CESR RF system operates at a very high value of $Y=$ 9.3 at $V_{\mathrm{c}}=1.86 \mathrm{MV}$ and $780 \mathrm{~mA}$ beam current and ii) one can not operate the system with optimal detuning for beam loading compensation because it becomes Robinson unstable above $500 \mathrm{~mA}$.

A conventional way to provide more stability to an RF system is to detune cavities off the optimal tuning angle $\psi_{0}$ determined from [6]

$$
\tan \psi_{0}=-Y \sin \varphi_{S}
$$

by a small amount $\chi$ called tuning offset. New tuning angle can then be calculated using the formula

$$
\tan \psi=-Y \frac{\sin \left(\varphi_{S}-\chi\right)}{\cos \chi}+\tan \chi .
$$

This relationship can be obtained from the phasor diagram presented in Figure 1.

For every value of beam current $I_{0}$ and tuning offset $\chi$ one can calculate tuning angle from (3) and then threshold current corresponding to the Robinson stability limit according to [7]

$$
I_{t h} \cong \frac{2 V_{c} \sin \varphi_{S}}{R / Q \cdot Q_{l} \sin 2 \psi} .
$$

Now we can define amplitude and phase stability margins as $A=\left(I_{\mathrm{th}}-I\right) / I$ and $P=\varphi_{\mathrm{s}}-\chi-\psi$ respectively. Superconducting cavities in CESR are typically running with the tuning offset of $\chi=-10^{\circ}$. This provides an amplitude stability margin of $2.2 \%$ and a phase stability margin of $4.7^{\circ}$ at $1 \mathrm{~A}$ beam current (Fig. 2 and 3).

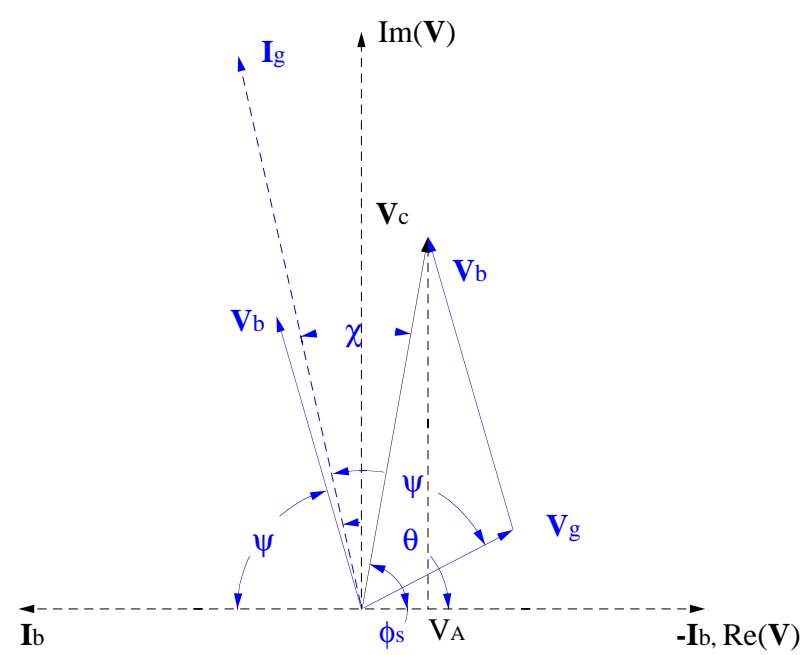

Figure 1: Phasor diagram for Robinson stability calculations. 


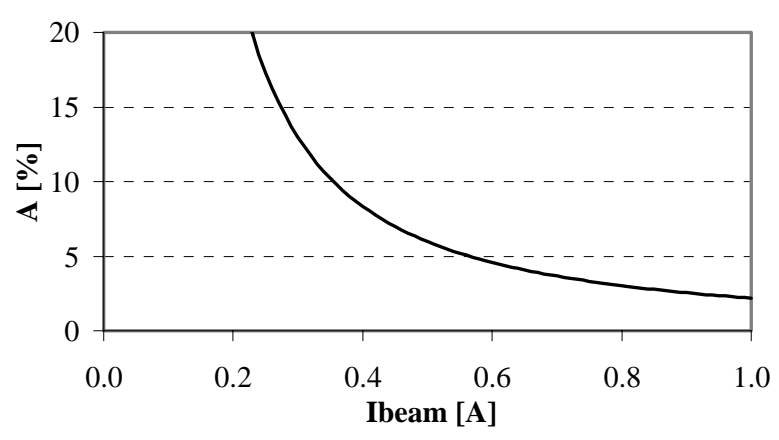

Figure 2: Dependence of the RF amplitude stability margin on the beam current in CESR.

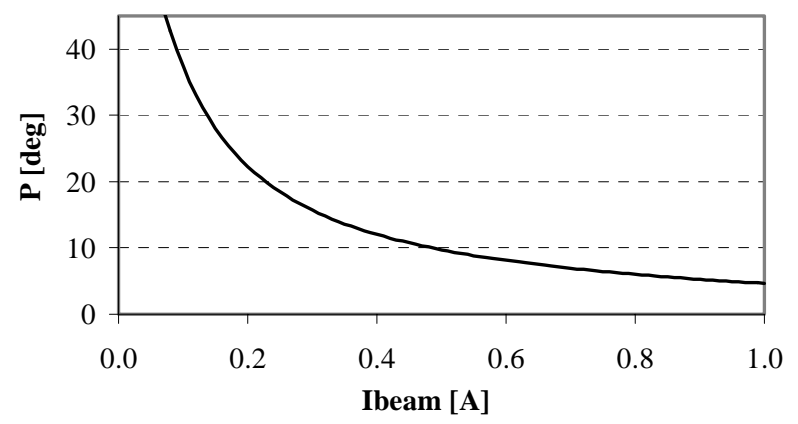

Figure 3: Dependence of the RF phase stability margin on the beam current in CESR.

An example of an unstable CESR RF system is shown in Figure 4. Due to malfunctioning of the W2 cavity tuning loop electronics the cavity was detuned off the optimal tuning angle to the wrong direction. As a result W2 cavity RF became Robinson unstable, RF tripped and the beam was lost. The figure presents following signals recorded by the CESR beamloss diagnostic system [8]: the cavity forward and reflected power, field and tuning angle error.

\section{RF PHASE CONTROL}

Under heavy beam loading conditions RF phase errors lead to uneven RF power demand between RF stations and additional mismatch. For example, $1^{\circ}$ phase error on one of the CESR cavities produces $21 \mathrm{~kW}$ change in power delivered to the beam by that cavity. So even though RF phase loop can regulate phase with error much smaller than one degree, slow thermal drifts in reference line, in the cable between cavity field probe and phase detector or in electronics can exceed one degree. In this case phase detector operating point shifts to a new position resulting in RF power misbalance between cavities.

Heavy beam loading provides us with means other than phase detectors to measure and adjust relative cavity phase by measuring RF power delivered to the beam and adjusting low-level phase shifters as proposed in [4]. Slow relative RF phase drift in CESR is measured to be up to $\pm 2^{\circ}$ over the course of several days. We wrote a short program, which periodically (once every HEP run)
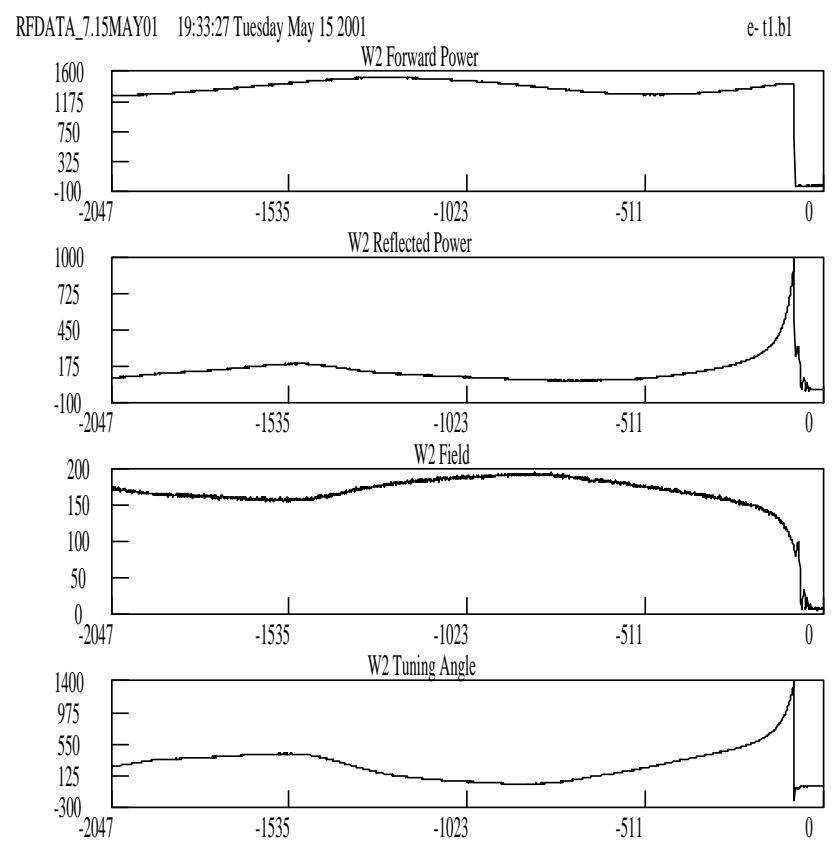

Figure 4: RF trip event caused by W2 cavity RF system becoming unstable. The vertical scales are in arbitrary units; the horizontal scale is time in CESR turns ( 1 turn = $2.56 \mu \mathrm{s})$.

measures RF power for each cavity and adjusts RF phase set points to keep a cavity RF power error relative to the average power per cavity within certain range (typically $\pm 10 \mathrm{~kW})$.

\section{MICROPHONICS}

There are two major sources of microphonic noise: cryogenic system and surrounding equipments such as mechanical vacuum pumps, water pumps, etc. Though microphonic noise is not considered normally a limiting factor for superconducting cavities with low loaded quality factor like $2.4 \times 10^{5}$ in the case of CESR, it can nevertheless present a problem under heavy beam loading conditions.

The very first superconducting cavity installed in CESR was E2. It replaced one of the old normal conducting cavities and was operated in a pair with the other (E1) normal conducting cavity. A low frequency vibration from the E1 cavity water pump caused very strong microphonic effect in the E2 cavity. We had to add heavy stiffeners to the cryostat to reduce parasitic amplitude and phase modulation to a tolerable level. The microphonic noise was drastically reduced when a superconducting cavity replaced the normal conducting one in the E1 position. Typical amplitudes of the parasitic RF phase an amplitude modulations are $0.5^{\circ}$ and $1 \%$. Amplitude of the parasitic modulation of a cavity resonant frequency is 25 $\mathrm{Hz}\left(1.4^{\circ}\right.$ on the tuning offset signal).

\section{RF CONTROLS FOR CESRC}

Let us consider in this section how converting CESR to operate at low energy [3] will effect requirements to RF 
regulating loops. As it is shown in [9], higher required RF voltage necessitates adding two more cavities. While RF voltage required by CESRc is high, RF power demand is very moderate: $160 \mathrm{~kW}$ at the energy of $2.5 \mathrm{GeV}, 90 \mathrm{~kW}$ at $1.89 \mathrm{GeV}$, and $40 \mathrm{~kW}$ at $1.55 \mathrm{GeV}$. These power levels hardly present a problem for RF power couplers of CESR superconducting cavities. However, low power demand at the high voltage presents a problem for RF regulation loops. A beam synchronous phase in these conditions is $88.3^{\circ}$ at $2.5 \mathrm{GeV}, 88.6^{\circ}$ at $1.89 \mathrm{GeV}$, and $88.8^{\circ}$ at 1.5 $\mathrm{GeV}$, meaning that bunches will pass the cavity gap very close to the RF wave null. Even slightest RF phase error could cause a large cavity mismatch and a big change of cavity voltage (in a case of operating with more than one cavity per klystron) and power delivered to the beam, which is unacceptable! The beam loading parameter in this case is extremely high: $34.5(2.5 \mathrm{GeV}), 40(1.89$ $\mathrm{GeV})$, and $48.7(1.55 \mathrm{GeV})$. As even one transmitter is more than adequate to supply necessary power, it was proposed [9] to operate four out of six cavities in a passive mode and use only one klystron to feed the two remaining active cavities.

The passive cavities will consume some amount of RF power because of the finite value of their loaded quality factor. To lower this consumption one would need to increase the cavity loaded $Q$ factor. It is reasonable to expect that we can increase it to $1 \times 10^{6}$. Then the additional required $\mathrm{RF}$ power is $180 \mathrm{~kW}(2.5 \mathrm{GeV}), 125$ $\mathrm{kW}(1.89 \mathrm{GeV})$ and $70 \mathrm{~kW}(1.55 \mathrm{GeV})$. To make the active cavities loaded by maximum beam current a matched load for the klystron their $Q_{l}$ will have to be increased to $2.7 \times 10^{5}(2.5 \mathrm{GeV}), 2.9 \times 10^{5}(1.89 \mathrm{GeV})$ and $3.2 \times 10^{5}(1.55 \mathrm{GeV})$. Because CESR cavities have fixed couplers we plan to use three-stub waveguide transformers [10] for coupling adjustment.

As one might expect, this significantly improves the situation. The synchronous phase becomes comparable with that of the present RF system: $79.4^{\circ}(2.5 \mathrm{GeV}), 79.7^{\circ}$ $(1.89 \mathrm{GeV}), 80.2^{\circ}(1.55 \mathrm{GeV})$. The same is true for the beam loading parameter: $5.4(2.5 \mathrm{GeV}), 5.6(1.89 \mathrm{GeV})$, $5.9(1.55 \mathrm{GeV})$.

As it was mentioned above we consider running two cavities out of one klystron. We ran the CESR RF system in this configuration on several occasions before (see, for example, [11]). Because our RF system control design is based on "classic" amplitude and phase analog feedback loops, it is impossible to implement true vector sum control of the total RF voltage. We used the so-called "master-slave" configuration of the cavity field regulation, when a cavity field signal from only one cavity ("master") is used in the feedback loop. The other cavity ("slave") passively follows by virtue of the cavities' similarity. Of course any difference in cavity couplings, positions, RF phase, non-equal power split by a magic T, etc. causes the field of the "slave" cavity to deviate form the set point. That is why the "master" is always the weaker cavity, i.e. the cavity with lower accelerating field limit. The RF phase loop was configured to regulate the sum of two RF phases. Having recognized shortcomings of the existing RF electronics, we are going to upgrade RF controls with the new I/Q feedback electronics based on digital signal processing. This will add more flexibility to the RF system and allow us to use vector sum control.

\section{CONCLUSIONS}

Steady-state stability analysis of the CESR RF system shows that tuning offset of $-10^{\circ}$ provides large enough stability margins to store beam currents up to $1 \mathrm{~A}$. Extremely heavy beam loading of superconducting cavities in CESR provides us with means to compensate slow RF phase variations, which are not regulated out by RF phase feedback loops. Microphonic noise, though noticeable, does not present a problem for our control electronics. A solution of very tight requirements to the RF regulation loops low energy operation of CESR was proposed in form of operating four out of six cavities in the passive mode. The new RF control electronics is under development for the RF system upgrade.

\section{REFERENCES}

[1] D. Rubin, et al., "CESR Status," this conference.

[2] S. Belomestnykh et al., "Running CESR at High Luminosity and Beam Current with Superconducting RF System," Proc. of the 7th EPAC, Vienna, Austria, June 2000, Vol. 3, pp. 2025-2027.

[3] D. Rice, et al., "Parameters for Low Energy Operation of CESR," this conference.

[4] K. Akai, "RF Issues for High Intensity Factories," Proc. of the 5th European Part. Accel. Conf., Barcelona, Spain, June 1996, Vol. 1, pp. 205-209.

[5] H. Padamsee, et al., "Design Challenges for High Current Storage Rings," Part. Accel., 40, pp. 14-41 (1992).

[6] P.B. Wilson, "Fundamental-Mode rf Design in $e^{+} e^{-}$ Storage Ring Factories," SLAC-PUB-6062 (1993).

[7] H. Padamsee, J. Knobloch, T. Hays, $R F$ Superconductivity for Accelerators, Wiley (1998).

[8] S. Henderson, "A Beamloss Diagnostic System for CESR," Proc. of the 1997 Part. Accel. Conf., Vol. 2, pp. 2029-2031.

[9] S. Belomestnykh, et al., "Superconducting RF System Upgrade for Short Bunch Operation of CESR," this conference.

[10] B. Dwersteg, "SC-Cavity Operation via WGTransformer." Proceedings of the 4th SRF Workshop, KEK Report 89-21, Vol. 2, pp. 593-604.

[11] S. Belomestnykh, et al., "Operating Experience with Superconducting RF at CESR and Overview of Other SRF Related Activities at Cornell University," Proc. of the 9th Workshop on RF Superconductivity, Santa Fe, NM, November 1999, Vol. 1, pp. 24-30. 